\title{
Investigations on the Spin-Hamiltonian Parameters and Local Structure of the Orthorhombic $\mathrm{Cu}^{2+}$ Center in $\mathrm{PbTiO}_{3}$ Crystal
}

\author{
Li ChaO-Ying*, Cheng Li-Bin and Zheng Xue-Mei \\ School of Physics and Electronic Information, Shangrao Normal University, Shangrao Jiangxi 334000, P.R. China
}

(Received September 14, 2014; in final form March 11, 2015)

The spin-Hamiltonian parameters (the $g$ factors $g_{i}$ and the hyperfine structure constants $A_{i}, i=x, y, z$ ) and local structure of the $\mathrm{Cu}^{2+}$ center in $\mathrm{PbTiO}_{3}$ are theoretically studied by using the perturbation formulae of these parameters for a $3 d^{9}$ ion in an orthorhombically elongated octahedra. The orthorhombic center is attributed to $\mathrm{Cu}^{2+}$ occupying the host $\mathrm{Ti}^{4+}$ site associated with a nearest-neighbouring oxygen vacancy $\mathrm{V}_{\mathrm{O}}$ along the $c$-axis, and the impurity $\mathrm{Cu}^{2+}$ off-center displacement $\Delta Z_{\mathrm{L}}(\approx 0.18 \AA)$ is smaller than that of the host $\mathrm{Ti}^{4+}$ site $\left(\Delta Z_{\mathrm{H}} \approx 0.3 \AA\right)$. Meanwhile, the planar $\mathrm{Cu}^{2+}-\mathrm{O}^{2-}$ bonds are found to experience the relative variation $\Delta R(\approx 0.098 \AA)$ along the $a$ - and $b$-axes, respectively, due to the Jahn-Teller effect and the size mismatching substitution of $\mathrm{Ti}^{4+}$ by $\mathrm{Cu}^{2+}$. The theoretical spin-Hamiltonian parameters based on the above defect structure agree well with the observed values.

DOI: $10.12693 /$ APhysPolA.127.1671

PACS: 76.30.Fc, 75.10.Dg, 71.70.Ch

\section{Introduction}

$\mathrm{PbTiO}_{3}$ as a member of ferroelectric perovskite family is a potential material with the technological applications in ferroelectric-volatile memory devices and piezoelectric actuators [1-3]. The physical and chemical properties of perovskites may be selectively changed by doping with transition-metal (TM) ions. For understanding the properties caused by these doping ions, many electron paramagnetic resonance (EPR) spectra of $\mathrm{PbTiO}_{3}$ doped with TM impurities have been made [4-8]. The EPR technique is a powerful tool to study the defect model and defect structure of paramagnetic impurity centers in crystals. These studies show that divalent and trivalent states of substitutional $3 d^{n}$ ions at $\mathrm{Ti}^{4+}$ site can be charge compensated by a nearest-neighbour oxygen vacancy $\mathrm{V}_{\mathrm{O}}$, and the microstructure of these $\mathrm{M}^{n+}-\mathrm{V}_{\mathrm{O}}$ centers has attracted the attention of many investigators [9-11]. Amongst these TM ions, $\mathrm{Cu}^{2+}\left(3 d^{9}\right)$ ion is a model system with a single $3 d$ hole, corresponding to only one ground state and one excited state under ideal octahedral crystal-fields. EPR studies for $\mathrm{Cu}^{2+}$ can provide important structural and electronic information of the doped materials and are of specific significance. For example, Warren et al. [12] performed EPR studies on $\mathrm{Cu}^{2+}$ doped in $\mathrm{PbTiO}_{3}$, and the spin-Hamiltonian (SH) parameters (the $g$ factors $g_{i}$ and the hyperfine structure constants $\left.A_{i}, i=x, y, z\right)$ were also measured. The observed $g$ factor $\left(g_{x} \approx 2.051, g_{y} \approx 2.065\right.$, and $g_{z} \approx 2.340$ [12]) of the orthorhombic center for $\mathrm{PbTiO}_{3}: \mathrm{Cu}^{2+}$ was very close to that $\left(g_{x} \approx 2.106, g_{y} \approx 2.076\right.$, and $\left.g_{z} \approx 2.381[13]\right)$ for the impurity $\mathrm{Cu}^{2+}$ ion doped in $\mathrm{LiNbO}_{3}$ with or-

\footnotetext{
* corresponding author; e-mail: cyli1962@gmail.com
}

thorhombic $\left[\mathrm{CuO}_{5}\right]^{8-}$ cluster. Therefore, there are good reasons to assume that the observed $\mathrm{SH}$ parameters of the orthorhombic center for $\mathrm{PbTiO}_{3}: \mathrm{Cu}^{2+}$ was due to the impurity $\mathrm{Cu}^{2+}$ substituting $\mathrm{Ti}^{4+}$ site with one nearest neighbour oxygen vacancy $\mathrm{V}_{\mathrm{O}}$ along the $c$ axis as the compensator similar as $\mathrm{LiNbO}_{3}: \mathrm{Cu}^{2+}[13]$.

Up to now, however, the above experimental SH parameters have not been theoretically explained, and the information about local structures of the impurity $\mathrm{Cu}^{2+}$ in $\mathrm{PbTiO}_{3}$ has not been obtained yet. Considering that microscopic mechanisms of the SH parameters and information of impurity local structures can be helpful to understand the properties of these TM ion doped materials, further theoretical investigations on these $\mathrm{SH}$ parameters and the local structures for the $\mathrm{Cu}^{2+}$ center in $\mathrm{PbTiO}_{3}$ are of scientific and technical significance. In this paper, the high (fourth) order perturbation formulae of the SH parameters for a $3 d^{9}$ ion under orthorhombically elongated octahedra are adopted for the analysis of the above $\mathrm{Cu}^{2+}$ center. Based on these studies, some useful information of defect structures for $\mathrm{Cu}^{2+}$ in $\mathrm{PbTiO}_{3}$ can be acquired.

\section{Calculations}

In $\mathrm{PbTiO}_{3}$ crystal, both the host $\mathrm{Pb}^{2+}$ and $\mathrm{Ti}^{4+}$ ions suffer large displacements from their corresponding oxygen planes by about 0.47 and $0.3 \AA$ at room temperature, resulting in a colossal tetragonality of $6.5 \%$ along with a $0.75 \mathrm{C} / \mathrm{m}^{2}$ spontaneous polarization $[14,15]$. When the impurity $\mathrm{Cu}^{2+}$ ion enters the lattice of $\mathrm{PbTiO}_{3}$, it may occupy the $\mathrm{Ti}^{4+}$ site due to similar ionic radius, despite of its significant charge mismatch. Since $\mathrm{Cu}^{2+}$ has less charge as compared with the replaced $\mathrm{Ti}^{4+}$, one nearest neighbour oxygen vacancy $\mathrm{V}_{\mathrm{O}}$ may occur along the $c$-axis as compensator, forming $\left[\mathrm{CuO}_{5}\right]^{8-}$ cluster. For the Jahn-Teller ion $\mathrm{Cu}^{2+}$, the planar ligands can suffer a relative bond length variation due to the Jahn-Teller effect 
via vibration interaction by compressing and stretching the $\mathrm{Cu}^{2+}-\mathrm{O}^{2-}$ bonds along the $a$ - and $b$-axes, respectively, which can bring forward moderate orthorhombic distortion to the impurity center. Meanwhile, the charge mismatching substitution of $\mathrm{Ti}^{4+}$ by $\mathrm{Cu}^{2+}$ may also lead to some modifications of the planar bond lengths. On the other hand, the impurity $\mathrm{Cu}^{2+}$ off-center displacement $\Delta Z_{\mathrm{L}}$ would be dissimilar to that $\left(\Delta Z_{\mathrm{H}} \approx 0.3 \AA[15]\right)$ for the host $\mathrm{Ti}^{4+}$ site.

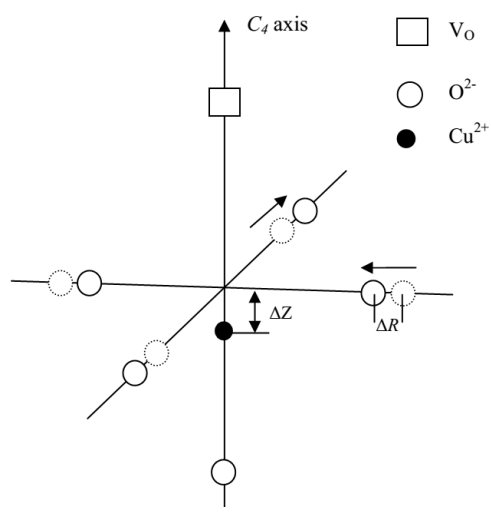

Fig. 1. Projective view of the impurity $\mathrm{Cu}^{2+}$ center in $\mathrm{PbTiO}_{3}$ crystal with one oxygen vacancies adjacent along $C_{4}$ axis.

As a result, the local structure of the impurity center can be described by the $\left[\mathrm{CuO}_{5}\right]^{8-}$ cluster and the impurity off-center displacement (characterized by $\Delta Z_{\mathrm{L}}$ ) and the relative planar bond length variation (characterized by $\Delta R$ ) (see Fig. 1). Consequently, the impurity-ligand bond lengths are divided into three groups, i.e., the planar ones labeled as " $R_{1}$ " and " $R_{2}$ " along the $a$ - and $b$-axes due to the bond length variation $\Delta R$ and the off-planar one labeled as " $R_{3}$ " along the $c$-axis. The angles between the planar bond lengths $R_{1}$ and $R_{2}$ and the $c$-axis are defined as $\theta_{1}$ and $\theta_{2}$, respectively. The local bond lengths and bond angles are determined as

$$
\begin{aligned}
& R_{1} \approx\left[\left(R_{\perp}-\Delta R\right)^{2}+\Delta Z_{\mathrm{L}}^{2}\right]^{1 / 2}, \\
& R_{2} \approx\left[\left(R_{\perp}+\Delta R\right)^{2}+\Delta Z_{\mathrm{L}}^{2}\right]^{1 / 2}, R_{3} \approx R_{\|}-\Delta Z_{\mathrm{L}}, \\
& \cos \theta_{1} \approx \Delta Z_{\mathrm{L}} / R_{1}, \quad \cos \theta_{2} \approx \Delta Z_{\mathrm{L}} / R_{2} .
\end{aligned}
$$

Thus, the impurity-ligand bonding lengths are unlike the host Ti-O distances $R_{\|} \approx 2.076 \AA$ and $R_{\perp} \approx 1.952 \AA[15]$ parallel and perpendicular to the $c$-axis for the center of the oxygen octahedron in the host $\mathrm{PbTiO}_{3}$.

For a $\mathrm{Cu}^{2+}\left(3 d^{9}\right)$ ion in orthorhombically elongated octahedra, its lower orbital doublet ${ }^{2} E_{\mathrm{g}}$ would be separated into two singlets ${ }^{2} A_{1 \mathrm{~g}}(\theta)$ and ${ }^{2} A_{1 \mathrm{~g}}^{\prime}(\varepsilon)$, with the latter lying lowest $[16,17]$. Meanwhile, the higher cubic orbital triplet ${ }^{2} T_{2 \mathrm{~g}}$ would be split into three singlets ${ }^{2} B_{1 \mathrm{~g}}(\zeta)$, ${ }^{2} B_{2 \mathrm{~g}}(\eta)$ and ${ }^{2} B_{3 \mathrm{~g}}(\xi)[16,17]$. Considering that the spinorbit parameter $\zeta_{p}^{0}\left(\approx 150 \mathrm{~cm}^{-1}\right.$ [18]) of ligand $\mathrm{O}^{2-}$ is much smaller than that $\zeta_{d}^{0}\left(\approx 829 \mathrm{~cm}^{-1}[19]\right)$ of the central ion $\mathrm{Cu}^{2+}$, the contributions to $\mathrm{SH}$ parameters due to the spin-orbit parameter of ligand via covalence effect is small and can be omitted, as in the conventional crystalfield theory. Then, the high order perturbation formulae of the SH parameters based on the conventional crystalfield model [18] containing merely the central ion orbital and spin-orbit coupling contributions can be reasonably adopted here. Thus, we have

$$
\begin{aligned}
& g_{x}=g_{\mathrm{s}}+\frac{2 k \varsigma}{E_{2}}+k \varsigma^{2} \frac{2 E_{3}-E_{1}-4 E_{2}}{E_{1} E_{2} E_{3}} \\
& +g_{\mathrm{s}} \varsigma^{2} \frac{4 E_{2}^{2} E_{3}^{2}-E_{1}^{2} E_{3}^{2}-E_{1}^{2} E_{2}^{2}}{2 E_{1}^{2} E_{2}^{2} E_{3}^{2}} \\
& -k \varsigma^{3}\left[\frac{E_{1}\left(E_{3}^{2}-E_{2}^{2}\right)+E_{3}\left(4 E_{2}^{2}-E_{1}^{2}\right)}{2 E_{1}^{2} E_{2}^{2} E_{3}^{2}}-\frac{E_{3}-E_{2}}{2 E_{2}^{2} E_{3} E_{4}}\right] \\
& +g_{s} \varsigma \frac{E_{1}^{2} E_{3}^{2}+2 E_{1}^{2} E_{2}^{2}-E_{1}^{2} E_{2} E_{3}+2 E_{2} E_{3}^{3}-2 E_{2}^{2} E_{3}^{2}-2 E_{1} E_{2}^{2} E_{3}}{4 E_{1}^{2} E_{2}^{2} E_{3}^{3}}, \\
& g_{y}=g_{\mathrm{s}}+\frac{2 k \varsigma}{E_{3}}+k \varsigma^{2} \frac{2 E_{2}-E_{1}-4 E_{3}}{E_{1} E_{2} E_{3}} \\
& +g_{\mathrm{s}} \varsigma^{2} \frac{4 E_{2}^{2} E_{3}^{2}-E_{1}^{2} E_{2}^{2}-E_{1}^{2} E_{3}^{2}}{2 E_{1}^{2} E_{2}^{2} E_{3}^{2}} \\
& -k \varsigma^{3}\left[\frac{E_{1}\left(E_{2}^{2}-E_{3}^{2}\right)+E_{2}\left(4 E_{3}^{2}-E_{1}^{2}\right)}{2 E_{1}^{2} E_{2}^{2} E_{3}^{2}}-\frac{E_{2}-E_{3}}{2 E_{2} E_{3}^{2} E_{4}}\right] \\
& +g_{s} \varsigma^{3} \frac{E_{1}^{2} E_{2}^{2}+2 E_{1}^{2} E_{3}^{2}-E_{1}^{2} E_{2} E_{3}+2 E_{2}^{3} E_{3}-2 E_{2}^{2} E_{3}^{2}-2 E_{1} E_{2} E_{3}^{2}}{4 E_{1}^{2} E_{2}^{3} E_{3}^{2}}, \\
& g_{z}=g_{\mathrm{s}}+\frac{8 k \varsigma}{E_{1}}+k \varsigma^{2} \frac{E_{1}-2 E_{2}-2 E_{3}}{E_{1} E_{2} E_{3}} \\
& +g_{\mathrm{s}} \varsigma^{2} \frac{4 E_{2}^{2} E_{3}^{2}-E_{1}^{2} E_{3}^{2}-2 E_{1}^{2} E_{2}^{2}}{4 E_{1}^{2} E_{2}^{2} E_{3}^{2}} \\
& -k \varsigma^{3} \frac{12 E_{1} E_{2} E_{3}-\left(E_{1}^{2}+4 E_{2} E_{3}\right)\left(E_{2}+E_{3}\right)}{2 E_{1}^{2} E_{2}^{2} E_{3}^{2}} \\
& +g_{s} \varsigma^{3} \frac{2 E_{2}^{2}+2 E_{3}^{2}-E_{1} E_{3}-E_{1} E_{2}}{4 E_{1} E_{2}^{2} E_{3}^{2}}, \\
& A_{x}=P\left[-\kappa-\frac{2}{7}+\left(g_{x}-g_{\mathrm{s}}\right)-\frac{3\left(g_{y}-g_{\mathrm{s}}\right)}{14}\right] \text {, } \\
& A_{y}=P\left[-\kappa-\frac{2}{7}+\left(g_{y}-g_{\mathrm{s}}\right)-\frac{3\left(g_{x}-g_{\mathrm{s}}\right)}{14}\right] \text {, } \\
& A_{z}=P\left[-\kappa+\frac{4}{7}+\left(g_{z}-g_{\mathrm{s}}\right)-\frac{6\left(g_{x}+g_{y}-2 g_{\mathrm{s}}\right)}{14}\right]
\end{aligned}
$$

Here $g_{\mathrm{s}}(\approx 2.0023)$ is the spin-only value. $k\left(\approx N^{2}\right)$ is the orbital reduction factor, characteristic of the covalence effect of the studied system. $\zeta$ and $P$ are, respectively, the spin-orbit coupling coefficient and the dipolar hyperfine structure parameter for the $3 d^{9}$ ion in crystals. Because of the covalence reduction effect for $3 d^{n}$ ions in crystals, we have $[20,21]$ :

$$
\varsigma \approx N^{2} \varsigma_{d}^{0}, \quad P \approx N^{2} P_{0}
$$

Here, for a free $\mathrm{Cu}^{2+}$ ion, the dipolar hyperfine structure parameter $P_{0}\left(\approx 388 \times 10^{-4} \mathrm{~cm}^{-1}[22]\right)$. The isotropic 
core polarization constants satisfies the relationship $\kappa \approx$ $-2 \chi /\left(3\left\langle r^{-3}\right\rangle\right)[23]$. Here $\chi$ is characteristic of the density of unpaired spins at the nucleus of the central ion, and $\left\langle r^{-3}\right\rangle$ is the expectation value of the inverse cube of the radial wave function of the $3 d$ orbital in crystals in terms of the related free-ion value $\left\langle r^{-3}\right\rangle_{0}$ multiplying the covalence factor $N$. From the data $\left\langle r^{-3}\right\rangle_{0} \approx 8.252$ a.u. [16] and $\chi \approx-3.12$ a.u. [23] for $\mathrm{Cu}^{2+}$ in some oxides, the value $\kappa \approx 0.29$ may be estimated and used for the studied system here.

The denominators $E_{i}(i=1-4)$ stand for the energy separations between the excited ${ }^{2} A_{1 \mathrm{~g}}(\theta),{ }^{2} B_{1 \mathrm{~g}}(\zeta)$, ${ }^{2} B_{2 \mathrm{~g}}(\eta)$ and ${ }^{2} B_{3 \mathrm{~g}}(\xi)$ and the ground ${ }^{2} A_{1 \mathrm{~g}}^{\prime}(\varepsilon)$ states, respectively. They are determined from the energy matrix for a $3 d^{9}$ ion under orthorhombic symmetry in terms of the cubic field parameter $D_{\mathrm{q}}$ and the orthorhombic field parameters $D_{\mathrm{s}}, D_{\mathrm{t}}, D_{\xi}$ and $D_{\eta}$ :

$$
\begin{aligned}
& E_{1} \approx 4 D_{\mathrm{s}}+5 D_{\mathrm{t}}, \quad E_{2} \approx 10 D_{\mathrm{q}}, \\
& E_{3} \approx 10 D_{\mathrm{q}}-3 D_{\mathrm{s}}+5 D_{\mathrm{t}}+3 D_{\xi}-4 D_{\eta}, \\
& E_{4} \approx 10 D_{\mathrm{q}}+D_{\mathrm{s}}+10 D_{\mathrm{t}}-3 D_{\xi}+4 D_{\eta} .
\end{aligned}
$$

From the superposition model [24] and the local geometrical relationship of the studied impurity center, the orthorhombic field parameters are determined as follows:

$$
\begin{aligned}
D_{\mathrm{s}} & =\frac{4}{7} \bar{A}_{2}\left(R_{0}\right)\left[\left(\frac{R_{0}}{R_{3}}\right)^{t_{2}}+\sum_{i=1,2}\left(3 \cos ^{2} \theta_{i}-1\right)\left(\frac{R_{0}}{R_{i}}\right)^{t_{2}}\right] \\
D_{\xi} & =\frac{2}{7} \bar{A}_{2}\left(R_{0}\right) \sum_{i=1,2}(-1)^{i} \sin ^{2} \theta_{i}\left(\frac{R_{0}}{R_{i}}\right)^{t_{2}}, \\
D_{\mathrm{t}} & =-\frac{8}{21} \bar{A}_{4}\left(R_{0}\right) \frac{1}{4} \sum_{i=1,2}\left(35 \cos ^{4} \theta_{i}-30 \cos ^{2} \theta_{i}\right. \\
& \left.+3-7 \sin ^{4} \theta_{i}\right)\left(\frac{R_{0}}{R_{i}}\right)^{t_{4}}, \\
D_{\eta} & =\frac{5}{42} \bar{A}_{4}\left(R_{0}\right) \sum_{i=1,2} \sin ^{2} \theta_{i}\left(7 \cos ^{2} \theta_{i}-1\right)\left(\frac{R_{0}}{R_{i}}\right)^{t_{4}} .
\end{aligned}
$$

Here $t_{2} \approx 3$ and $t_{4} \approx 5[10,14,25-28]$ are the powerlaw exponents due to the dominant ionic nature of the bonds. $\bar{A}_{2}\left(R_{0}\right)$ and $\bar{A}_{4}\left(R_{0}\right)$ are the intrinsic parameters, with the reference bond length $R_{0}$ taken as the average of the metal-ligand distances (i.e., $R_{0}=\left(R_{\|}+\right.$ $\left.\left.R_{\perp}\right) / 3 \approx 1.993 \AA\right)$. For $3 d^{n}$ ion in octahedral crystalfields, the relationships $\bar{A}_{4}\left(R_{0}\right) \approx(3 / 4) D_{\mathrm{q}}$ and the ratio $\bar{A}_{2}\left(R_{0}\right) / \bar{A}_{4}\left(R_{0}\right)$ is in the range $8-12$ for $3 d^{n}$ ions in many crystals $[10,22,26-28]$, we take the average value $\bar{A}_{2}\left(R_{0}\right) \approx 10 \bar{A}_{4}\left(R_{0}\right)$ here. Thus, the $\mathrm{SH}$ parameters (especially the axial and perpendicular anisotropies $\Delta g=g_{z}-\left(g_{x}+g_{y}\right) / 2$ and $\left.\delta g=g_{y}-g_{x}\right)$ are correlated to the orthorhombic field parameters and hence to the local structure of the impurity center. For the studied orthorhombic $\left[\mathrm{CuO}_{5}\right]^{8-}$ cluster here, the cubic field parameter $D_{\mathrm{q}} \approx 820 \mathrm{~cm}^{-1}$ and the covalence factor $N \approx 0.80$ can be obtained from the tetragonal $\mathrm{Cu}^{2+}$ center in $\mathrm{PbTiO}_{3}: \mathrm{Cu}^{2+}$ based on the EPR analysis [10].

\section{TABLE I}

The calculated and experimental spin-Hamiltonian $g$ factors and the hyperfine structure constants (in $10^{-4} \mathrm{~cm}^{-1}$ ) for $\mathrm{PbTiO}_{3}: \mathrm{Cu}^{2+}$.

\begin{tabular}{c|c|c|c|c|c|c}
\hline \hline & $g_{x}$ & $g_{y}$ & $g_{z}$ & $A_{x}$ & $A_{y}$ & $A_{z}$ \\
\hline Cal. & 2.0547 & 2.0650 & 2.3336 & 7.991 & 10.874 & -116.457 \\
Exp. [12] & 2.051 & 2.065 & 2.340 & 7.74 & 9.34 & 112.05
\end{tabular}

TABLE II

The local structure bond lengths $R_{1}$ and $R_{2}$ and $R_{3}$ (in $\AA$ ), the angles $\theta_{1}$ and $\theta_{2}$ (in ${ }^{\circ}$ ), the cubic field parameter $D_{\mathrm{q}}$ and the orthorhombic field parameters $D_{\mathrm{s}}, D_{\mathrm{t}}, D_{\xi}$ and $D_{\eta}\left(\right.$ in $\left.\mathrm{cm}^{-1}\right)$ for $\mathrm{Cu}^{2+}$ center in $\mathrm{PbTiO}_{3}$.

\begin{tabular}{c|c|c|c|c|c|c|c|c|c}
\hline \hline$R_{1}$ & $R_{2}$ & $R_{3}$ & $\theta_{1}$ & $\theta_{2}$ & $D_{\mathrm{q}}$ & $D_{\mathrm{s}}$ & $D_{\mathrm{t}}$ & $D_{\xi}$ & $D_{\eta}$ \\
\hline 1.904 & 2.099 & 1.813 & 84.6 & 85.1 & 820 & -2640 & -115 & -606 & -32
\end{tabular}

Thus, there are only two unknown parameters, i.e., the impurity $\mathrm{Cu}^{2+}$ displacement $\Delta Z$ along the $c$-axis due to electrostatic repulsion of the $\mathrm{V}_{\mathrm{O}}$ and the planar bond length variation $\Delta R$ along the $a$ - and $b$-axes in Eq. (1) of the $\mathrm{SH}$ parameters. Substituting these parameters into Eq. (1) and fitting the calculated SH parameters to the experimental values, one can obtain

$$
\Delta Z \approx 0.18 \AA \text { and } \Delta R \approx 0.098 \AA .
$$

The corresponding theoretical results are collected in Table I. Meanwhile, the obtained local structural parameters $R_{1}, R_{2}, R_{3}$, the angles $\theta_{1}, \theta_{2}$ and the orthorhombic field parameters (i.e., $D_{\mathrm{s}}, D_{\mathrm{t}}, D_{\xi}$ and $D_{\eta}$ ) are listed in Table II.

\section{Discussion}

It can be seen from Table I that the calculated $\mathrm{SH}$ parameters for the $\mathrm{Cu}^{2+}$ center in $\mathrm{PbTiO}_{3}$ based on the local structure distortion obtained in this work show reasonable agreement with the experimental data. Thus, the defect model of $\mathrm{PbTiO}_{3}: \mathrm{Cu}^{2+}$ are therefore confirmed and the defect structure data (i.e., the impurity off-center displacement $\Delta Z_{\mathrm{L}}$; the planar bond length variation $\Delta R$ ) are obtained from the calculations.

(1) The experimental orthorhombic SH parameters for $\mathrm{PbTiO}_{3}: \mathrm{Cu}^{2+}$ are conveniently described by the axial and perpendicular anisotropies $\Delta g$ and $\delta g$. According to Eqs. (1), (2) and (5), the anisotropies arise mainly from the axial (i.e., the crystal-field parameters $D_{\mathrm{s}}$ and $D_{\mathrm{t}}$ due to the $\mathrm{V}_{\mathrm{O}}$ and the impurity off-center displacement $\Delta Z_{\mathrm{L}}$ ) and perpendicular (i.e., the $D_{\xi}$ and $D_{\eta}$ due to the planar bond length variation $\Delta R$ ) orthorhombic distortions of the ligand octahedron. Thus, the observed $\Delta g(\approx 0.282)$ and $\delta g(\approx 0.014)$ can also be reasonably attributed to the impurity off-center displacement $\Delta Z_{\mathrm{L}}(\approx 0.18 \AA)$ due to the electrostatic repulsion of the effectively positive $\mathrm{V}_{\mathrm{O}}$ and the planar bond length variation $\Delta R(\approx 0.098 \AA)$ due to the Jahn-Teller and size mismatch effect. Interestingly, similar impurity off-center 
displacements $(\approx 0.15-0.3 \AA)$ were also reported for various transition-metal ions (e.g., $\mathrm{Pt}^{3+}, \mathrm{Cu}^{2+}, \mathrm{Ru}^{3+}, \mathrm{Ni}^{3+}$ ) on the octahedral $\mathrm{Ti}^{4+}$ site in $\mathrm{PbTiO}_{3}$ based on the EPR analysis $[10,18,29,30]$.

(2) From Table I, one can see the absolute values of hyperfine structure constants $A_{i}(i=x, y, z)$ are in good agreement with the experimental findings and the signs for the calculated $A_{z}$ is negative, but the observed values given by Warren et al. are positive [12]. Actually, experimental determination of the signs of hyperfine structure constants for $3 d^{n}$ ions in crystals is very difficult, many experiments give them as absolute ones. The signs of the hyperfine structure constants $A_{i}$ suggested here are the same as those for $\mathrm{Cu}^{2+}$ doped in many crystals based on the EPR analysis [13, 22, 26] and can be regarded as reasonable. In addition, previous studies of hyperfine structure constants for $\mathrm{Cu}^{2+}$ ions in various crystals suggest that the constant $\kappa(\approx 0.25-0.35)[10,13,21,22]$. The value of $\kappa(\approx 0.29)$ for $\mathrm{PbTiO}_{3}: \mathrm{Cu}^{2+}$ obtained in this work lies within the range and can be considered to be suitable.

\section{Conclusion}

The orthorhombic $\mathrm{Cu}^{2+}$ center in $\mathrm{PbTiO}_{3}$ can be described as the substitutional $\mathrm{Cu}^{2+}$ on the $\mathrm{Ti}^{4+}$ site in $\mathrm{PbTiO}_{3}$, associated with one nearest neighbour $\mathrm{V}_{\mathrm{O}}$ along the $c$-axis. Based on this model assumption, the planar bond lengths are found to suffer the relative variation of about $0.098 \AA$ by compressing and stretching the $\mathrm{Cu}^{2+}$ $\mathrm{O}^{2-}$ bonds along the $a$ - and $b$-axes, respectively, due to the Jahn-Teller effect and the charge mismatching substitution. In addition, the impurity $\mathrm{Cu}^{2+}$ off-center displacement $\Delta Z_{\mathrm{L}}(\approx 0.18 \AA)$ is different from the host $\Delta Z_{\mathrm{H}}(\approx 0.30 \AA) \mathrm{Ti}^{4+}$ ion.

\section{Acknowledgments}

This work was financially supported by the Chinese Natural Science Foundation (Grant Nos. 11365017, and 11465015).

\section{References}

[1] M.E. Lines, A.M. Glass, em Principles and Applications of Ferroelectric and Related Materials, Oxford University Press, Oxford 2001

[2] J.V. Tol, K.P. Dinse, H. Kungl, M.J. Hoffmann, Appl. Magn. Reson. 36, 297 (2009).

[3] T.Y. Chen, S.Y. Chu, R.C. Chang, C.K. Cheng, C.S. Hong, H.H. Nien, Sens. Actuat. A 134, 452 (2007).

[4] V.V. Laguta, M.D. Glinchuk, I.P. Bykov, Yu.L. Maksimenko, J. Rosa, L. Jastrabík, Phys. Rev. B 54, 12353 (1996).

[5] V.V. Laguta, T.V. Antimirova, M.D. Glinchuk, I.P. Bykov, J. Rosa, M. Zaritskii, L. Jastrabik, J. Phys. Condens. Matter 9, 10041 (1997).
[6] D.J. Keeble, Z. Li, E.H. Poindexter, J. Phys. Condens. Matter 7, 6327 (1995).

[7] D.J. Keeble, E.H. Poindexter, G.J. Gerardi, Appl. Spectrosc. 51, 117 (1997).

[8] H. Meštrić, R.A. Eichel, T. Kloss, K.P. Dinse, So. Laubach, St. Laubach, P.C. Schmidt, K.A. Schönau, M. Knapp, H. Ehrenberg, Phys. Rev. B 71, 134109 (2005).

[9] R.A. Eichel, M.D. Drahus, P. Jakes, E. Erunal, E. Erdem, S.K.S. Parashar, H. Kungl, M.J. Hoffmann, Mol. Phys. 107, 1981 (2009).

[10] X.X. Wu, W.C. Zheng, S. Tang, Z. Naturforsch. 59a, 47 (2004).

[11] H. Meštrić, R.A. Eichel, K.P. Dinse, A. Ozarowski, J.V. Tol, L.C. Brunel, J. Appl. Phys. 96, 7440 (2004).

[12] W.L. Warren, B.A. Tuttle, E.C. Rong, G.J. Gerardi, E.H. Poindexter, J. Am. Ceram. Soc. 80, 680 (1997).

[13] M.Q. Kuang, S.Y. Wu, H.M. Zhang, Optick 123 1601 (2012).

[14] S.Y. Wu, H.M. Zhang, G.D. Lu, J.S. Yao, Z. Naturforsch. 62a, 338 (2007).

[15] M.J. Haun, E. Furman, S.J. Jang, H.A. McKinstry, L.E. Cross, J. Appl. Phys. 62, 3331 (1987).

[16] A. Abragam, B. Bleaney, Electron Paramagnetic Resonance of Transition Ions, Clarendon Press, Oxford 1970.

[17] A.S. Chakravarty, Introduction to the Magnetic Properties of Solids, Wiley-Interscience, New York 1980.

[18] S.Y. Wu, X.Y. Gao, J.Z. Lin, Q. Fu, J. Alloys Comp. 424, 55 (2006).

[19] J.S. Griffith, The Theory of Transition-Metal Ions, Cambridge University Press, London 1964.

[20] C.Y. Li, X.M. Zheng, Acta Phys. Pol. A 125, 73 (2014).

[21] W.Q. Yang, W.C. Zheng, P. Su, H.G. Liu, Cryst. Res. Technol. 45, 1132 (2010).

[22] H.N. Dong, S.Y. Wu, P. Li, Phys. Status Solidi B 241, 1935 (2004)

[23] B.R. McGarvey, J. Phys. Chem. 71, 51 (1967).

[24] D.J. Newman, B. Ng, Rep. Prog. Phys. 52, 699 (1989).

[25] H.N. Dong, Z. Naturforsch. 60a, 615 (2005).

[26] H.M. Zhang, S.Y. Wu, M.Q. Kuang, Z.H. Zhang, J. Phys. Chem. Solids 73, 846 (2012).

[27] W.C. Zheng, D.T. Zhang, P. Su, H.G. Liu, Spectrochim. Acta A 81, 548 (2011)

[28] W.L. Feng, L. He, W.Q. Yang, W.C. Zheng, Mol. Phys. 107, 2293 (2009).

[29] S.Y. Wu, Q. Fu, J.S. Yao, H.M. Zhang, Radiat. Eff Defects Solids 162, 627 (2007).

[30] B.T. Song, S.Y. Wu, M.Q. Kuang, X.F. Hu, Optik 124, 2493 (2013). 